Journal of Psychopharmacology

http://jop.sagepub.com/

Carryover effects to addiction-associated stimuli in a group of marijuana and cocaine users

Dinkar Sharma and Sharon Money

J Psychopharmacol 2010 24: 1309 originally published online 25 November 2009

DOI: $10.1177 / 0269881109350079$

The online version of this article can be found at:

http://jop.sagepub.com/content/24/9/1309

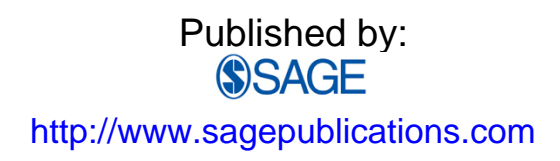

On behalf of:

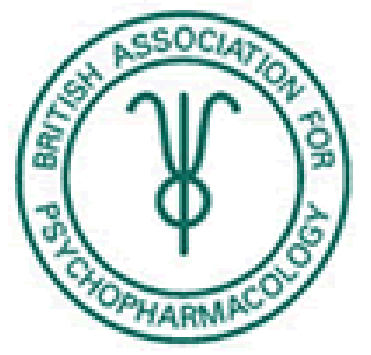

British Association for Psychopharmacology

Additional services and information for Journal of Psychopharmacology can be found at:

Email Alerts: http://jop.sagepub.com/cgi/alerts

Subscriptions: http://jop.sagepub.com/subscriptions

Reprints: http://www.sagepub.com/journalsReprints.nav

Permissions: http://www.sagepub.com/journalsPermissions.nav

Citations: http://jop.sagepub.com/content/24/9/1309.refs.html 


\title{
Carryover effects to addiction-associated stimuli in a group of marijuana and cocaine users
}

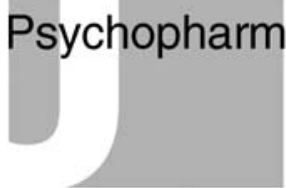

\author{
Dinkar Sharma and Sharon Money
}

Journal of Psychopharmacology

24(9) 1309-1316

(C) The Author(s) 2010

Reprints and permissions:

sagepub.co.uk/journalsPermissions.nav DOI: $10.1177 / 0269881109350079$

jop.sagepub.com

(s)SAGE

\begin{abstract}
Addiction has been characterized as an attentional bias towards drug-related cues. In two experiments we investigate the effects of non-words that have been associatively trained to addiction-related images in a group of marijuana and cocaine users. These associated non-words were presented along with unstudied non-words in a subsequent addiction Stroop task. Results indicate a slowdown in responding to the colour of non-words that were paired with cocaine-related images compared with non-cocaine related images. The slowdown was also characterized as a carryover effect, with the largest effect occurring on trials following the addiction-associated non-word. No effects were found for marijuana images associated with non-words.
\end{abstract}

Keywords

attention, bias, marijuana, cocaine, stroop, conditioning

\section{Introduction}

The study of marijuana and cocaine are important areas of research in their own rights because of their link to emotional disorders, crime, and the direct and indirect effects they can have on minors. Marijuana studies are important because marijuana use is a precursor for later cocaine use (Perry and Mandell, 1995) and because marijuana is often seen as a relatively 'harmless' drug. Misconceptions about the seriousness of marijuana addiction include the belief that marijuana addiction prevalence is low, that marijuana addiction occurs primarily in a poly-drug dependence context, and that marijuana addicts can easily stop their drug-taking (Budney et al., 1998). Furthermore, marijuana use is not directly associated with a substantial functional impairment whereas the seriousness of cocaine addiction is more readily noted (Budney et al., 1998; Lyvers, 1998). To date, few if any studies have used one paradigm to investigate both types of addiction; however, individual studies suggest that memory-associated drug cues play a large role in maintaining drug addiction behaviour (Ames and Stacy, 1998) by inducing physiological arousal (Lyvers, 1998).

The processing of marijuana stimuli by addicts, and in particular how such stimuli affect selective attention, has been investigated using the modified Stroop (1935) task. In the Addiction Stroop task, participants respond to the colour in which addiction and neutral words are presented whilst ignoring the meaning of the word. Typically, addictionrelated words take longer to colour-name than neutral words (for a review see Cox et al., 2006; Field and Cox, 2008). This interference or attentional bias (expressed as a difference in reaction time (RT) between addiction-related and neutral words) is thought to reflect the fact that although participants try to ignore the word's meaning, they are unable to do so and as a result attentional resources are redirected from colour naming to processing the relevance of the addiction word.

The addiction Stroop task has been used to investigate attentional bias to addiction-related stimuli across a number of addiction groups including smoking (Cane et al., 2009; Munafo et al., 2003; Waters et al., 2003a,b), alcohol (Albery et al., 2006; Sharma et al., 2001), and gambling (Boyer and Dickerson, 2003). Attentional bias has been implicated to have a causal role in maintaining substance use. For example, it has been related to the risk of subsequent relapse in smokers (Waters et al., 2003b), cocaine users (Carpenter et al., 2006), and alcohol users (Cox et al., 2002, 2007). Although much of the earlier work focussed on processing that took place during stimulus presentation, there is now growing evidence from the emotional and addiction Stroop tasks that interference can take place not only during stimulus presentation but can also carryover onto subsequent trials (Cane et al, 2009; Phaf and Kan, 2007, Waters et al., 2003a, 2005). The mechanism underlying this carryover effect is not known; however, Waters et al. (2005) have postulated rumination, difficulty with disengaging or switching attention, and initiation of conditioned responses as possible explanations.

The interpretation of the interference (both the immediate and carryover effects) found in the addiction Stroop task is

Department of Psychology, Keynes College, University of Kent, Kent, UK.

\section{Corresponding author:}

Dinkar Sharma, Department of Psychology, Keynes College, University of Kent, Canterbury, Kent CT2 7NP, UK. Email: d.sharma@kent.ac.uk 
made more difficult due to potential lexical confounds. Larsen et al. (2006) have recently argued that in the emotional Stroop task part of the interference could be accounted for by differences in the lexical properties of emotionally negative and neutral words (e.g. word frequency, neighbourhood size). The possibility that these lexical confounds could contribute to the carryover effect cannot be ruled out. Given the inherent difficulty with matching two sets of emotional or even addiction-related words, it suggests that alternative methods are required to study the interference produced from either emotional or addiction-related stimuli. One suggestion to overcome such confounds is to use non-words that have been randomly selected to be conditioned to emotional or neutral pictures (Richards and Blanchette, 2004). Pairing the two events (non-words and emotional stimulus) results in the non-word acquiring an emotional valence through association. Richards and Blanchette (2004) used this method to investigate the effects of associating emotional pictures to non-words. They showed that after learning the association, the emotionally associated non-words took longer to colour name as compared with non-words associated to neutral pictures, and that this interference was larger in a 'high anxious' group.

Previous research in addiction has used conditioning paradigms to show that addiction-related stimuli can act as unconditioned stimuli and elicit unconditioned responses (e.g. Robinson and Berridge, 1993). It has also been shown that repeatedly pairing an unconditioned stimulus with a conditioned stimulus can elicit conditioned responses such as craving (Carter and Tiffany, 2001; Dols et al., 2000) and physiological arousal (Lazev et al., 1999). Cues that have been associated to substance use can also affect attentional processes and are thought to be moderated by the expectancy that the substance will be available to use (Field and Cox, 2008; Hogarth and Duka, 2006).

The main aim of the present studies was to extend the associative-learning paradigm to investigate the effects of non-words that had been associated to addiction-related pictorial stimuli. One advantage of using non-words is that they have no prior links to addiction, and thus meaning can be brought under experimental control. Furthermore, if conditioning is successfully established, this shows the power of addiction to mediate its effects through non-addiction-related cues. Using addiction-associated non-words that are two steps removed from the unconditioned stimulus will provide strong support for this mediation process. After the initial learning phase, a test phase involving the addiction Stroop task was used. The non-words shown during the learning phase were repeated during the test phase in two separate blocks (addiction or neutral block). Each block mixed studied and unstudied non-words. The studied non-words were mixed pseudorandomly such that each studied non-word was followed by six unstudied non-words. This was done to enable us to observe carryover effects (see Cane et al., 2009; Waters et al., 2003a).

\section{Experiment 1}

In Experiment 1 marijuana users were trained with marijuana pictures as the unconditioned stimulus and non-words as the conditioned stimulus. Consistent with Cane et al. (2009) and Field et al. (2006), marijuana smokers were expected to show longer response latencies to respond to the colour of non-words that had been associated to marijuana images than to non-words that had been associated to neutral images. Furthermore, because of the increase in attentional hold that users exhibit to marijuana cues, it was predicted that the effects from the marijuana-associated non-words would lead to a carryover effect. This carryover effect should be observed as increased RTs on subsequent trials that included unstudied non-words that were mixed with marijuanaassociated non-words than with neutral associated nonwords.

\section{Method \\ Participants}

The sample consisted of 52 participants between the ages of 17 and 60 with a mean age of 31 . Thirty-eight of these were male and both sexes were divided equally across two groups (marijuana users and non-users). All participants were recruited through social networks and were known to be marijuana smokers or non-users. Half of the participants were university students. All participants were treated in accordance with the ethical standards of the British Psychological Association and ethical approval was obtained from the Department of Psychology ethics committee. Twenty-seven participants reported to be regular marijuana users and 25 participants indicated that they were not marijuana users. Of the marijuana users, 12 reported to be using at least once per week, and 15 reported using once per month or less. Nine had been using marijuana between zero and four years, and 18 participants indicated that they had been using for over four years.

\section{Design}

The design formed a $2 \times 2 \times 7$ mixed factorial design. The between-participant factor was addiction group, which had two levels: marijuana user or non-marijuana user. Nonword type was a within-participant factor that had two levels: addiction associated or neutral associated non-word. The third independent variable was position, that had seven levels: position 1, which was occupied by a non-word from the learning phase, and positions $2-7$, which were non-studied non-words. The dependent variable was reaction times for colour-naming.

\section{Materials}

The addiction Stroop task was presented using a 15-inch screen Toshiba laptop computer using an E-Prime program. A standard E-Prime response box was used to make the colour-naming response. An Olympus Camedia C-460 Zoom digital camera was used to create the five neutral pictures. Ten coloured pictures $(640 \times 480$ pixels $)$ were used for the conditioning phase: five marijuana-related images downloaded from the internet (marijunaaddiction.com), and five marijuana-unrelated images that were matched by ensuring 


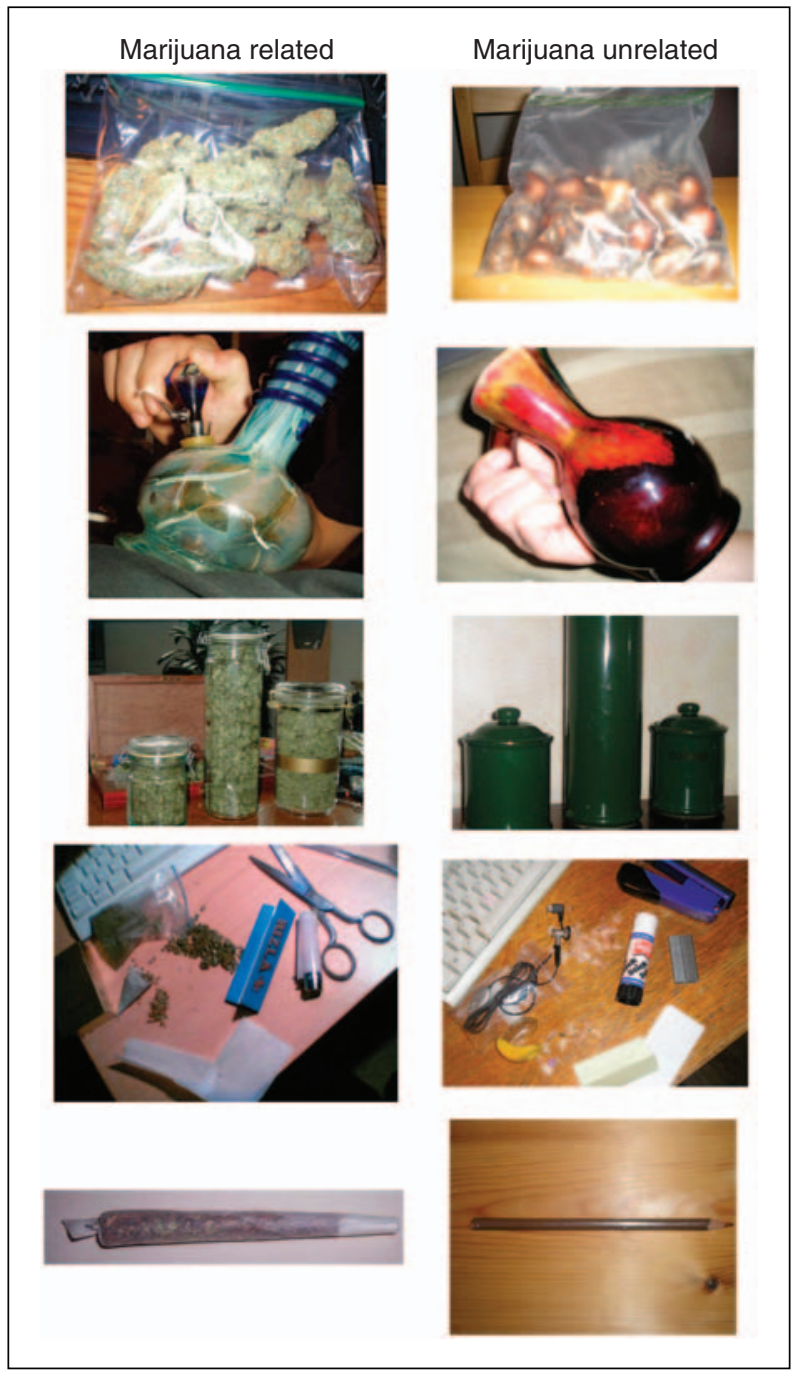

Figure 1. Pictures used to condition non-words in Experiment 1 (marijuana).

that the number, the general shape and size of the objects were similar (see Figure 1). A pool of 105 five-letter pronounceable non-words were selected from the ARC nonwords database, (Rastle et al., 2002), e.g. nease, qrinz, herze, ruque, repes, and were presented in lower case size 48 Arial font.

\section{Procedure}

From the initial pool of 105 non-words and for each participant, 10 non-words were randomly selected to be used in the learning phase (five randomly selected to be associated to the marijuana pictures and five to the neutral pictures). A total of 35 non-words were randomly selected to be used during practice to become familiar with the addiction Stroop task. The remaining 60 non-words were used during the addiction Stroop task (presented at positions 2-7) along with the 10 non-words shown during the learning phase (presented at position 1).

\section{Learning phase}

Participants were shown 10 coloured images that appeared on screen one at a time, paired with a non-word. Five of the pictures were marijuana related and five were neutral images. Each picture and non-word pairing stayed on the screen for five seconds and were presented three times in random order. In deciding the number of trials to use we took into account the balance between conditioning and habituation. Our judgement was that we did not want to risk overexposing participants to the pictures that could lead to habituation. This is why we initially used a relatively small number of learning trials (minimum of 30 learning trials). However, participants could receive additional learning trials (in groups of 30 trials) until they reached criterion ( $100 \%$ correct on the memory task).

Participants were instructed to learn which non-words were paired with addiction pictures, and which were paired with neutral pictures. A memory test followed that consisted of presenting the non-words without the pictures, one at a time, with participants responding by pressing one of two buttons as to whether each non-word had appeared with a marijuana image or a neutral image. If participants scored $100 \%$ on the memory test they proceeded to the practice phase. If they made any errors they repeated the learning phase until they reached criterion. On average the marijuana users did not differ in the number of trials they received during the learning phase $(M=58.89$ trials, $S E=6.50)$ compared with the non-marijuana users $(M=60.0$ trials, $S E=9.49), t(50)=0.098, p>0.9$.

\section{Practice phase}

During practice, 35 non-words were presented randomly one at a time in one of four colours: red, blue, yellow or green. Participants were instructed to use their middle and index fingers on each hand to make their responses and to respond to the colour of the ink of the non-words. They were asked to make their responses as quickly and accurately as possible. All non-words stayed on the screen until a response was made and the next trial appeared immediately after a response was made.

\section{Addiction Stroop phase}

For the addiction Stroop phase, the general procedure and instructions were identical to the practice phase. During the addiction Stroop task the 70 non-words not used during practice were presented six times each, giving a total of 420 trials. Participants completed two sessions. One session of 210 trials involved presenting the marijuana-associated non-words mixed with unstudied non-words and the other session mixed neutral-associated non-words with unstudied non-words. There was a short break between the two sessions and the order was counterbalanced across participants. Within each session the non-words were presented in a pseudorandom sequence, with the studied non-words (non-words shown during the learning phase) always appearing at position 1 followed by six trials of unstudied non-words (Positions 2-7).

Finally, after the addiction Stroop task, the memory test given during the learning phase was repeated to check if 
participants remembered the associations formed during the learning phase.

\section{Results and discussion}

\section{Analysis of addiction Stroop task}

The analysis was performed on the mean correct RT scores. Extreme scores of less than $300 \mathrm{~ms}$ and greater than $4000 \mathrm{~ms}$ were removed. The mean scores were analysed using a threeway mixed analysis of variance (ANOVA), with Position (1-7) and Non-word Type (marijuana-associated, nonmarijuana-associated) as within-subject factors, and Group (marijuana users, control non-marijuana users) as a between-subjects factor.

The main finding is that marijuana-associated non-words do not differ from non-marijuana-associated non-words when they are shown in an addiction Stroop task. The analysis revealed no significant main or interaction effects (all $F<1.8, p>0.1$ ). The marijuana users group ( $862 \mathrm{~ms}$ ) did not differ significantly from the non-user group ( $884 \mathrm{~ms}$ ) $F(1,50)=0.23, M S E=383920, p=0.631$. The main effect of Non-word Type indicated that the marijuana-associated nonwords $($ mean $=869 \mathrm{~ms}$ ) did not differ from the non-marijuana-associated non-words (mean $=877 \mathrm{~ms}) F(1,50)=1.14$, $M S E=8984, p=0.29$. There was also no interaction between Group and Non-word Type, $F(1,50)=1.26, M S E=8984$, $p=0.27$, or between Group, Non-word Type and Position, $F(6,300)=1.72, M S E=3816, p=0.12$ (see Figure 2).

Further analysis explored whether the effects of attentional bias might have been attenuated during the course of the Stroop task. We divided each session into two halves (105 trials in each half). An ANOVA included Block (first half, second half) as an additional factor (including Position, Nonword Type, and Group as other factors). This showed that there was a main effect of Block $F(1,50)=21.55, p<0.001$ ) with faster RTs in the first half $(858 \mathrm{~ms})$ than in the second half $(888 \mathrm{~ms})$. All other main and interaction effects were not significant $(F<1.74, p>0.1)$. The difference between the two Non-word Type conditions (marijuana-associated RT minus non-marijuana-associated non-word RT) tended to be less negative in the first half $(-1 \mathrm{~ms})$ than in the second half $(-95 \mathrm{~ms}), F(1,50)=1.43, p=0.24$.

We also explored whether there were any effects within those participants who scored $100 \%$ in the final memory test. Restricting analysis to this subgroup ( $N=13$ non-users; $N=16$ users) revealed no significant main or interaction effects (all $F<1.35, p>0.24$ ). In general these two additional analyses do not support an attentional bias to marijuanaassociated non-words.

Overall error rates were low $(3.3 \%)$. Error rates were analysed using a three-way mixed ANOVA, with Position (1-7) and Non-word Type (marijuana-associated, non-marijuanaassociated) as within-subject factors, and Group (marijuana users, control non-marijuana users) as a between-subjects factor. This analysis revealed only one significant effect, a two-way interaction, Non-word Type $\times$ Position, $F(1,50)=3.04, M S E=0.001, p<0.01$. Simple main effect of Non-word Type indicated more errors for non-marijuanaassociated non-words $(4.5 \%)$ than marijuana-associated

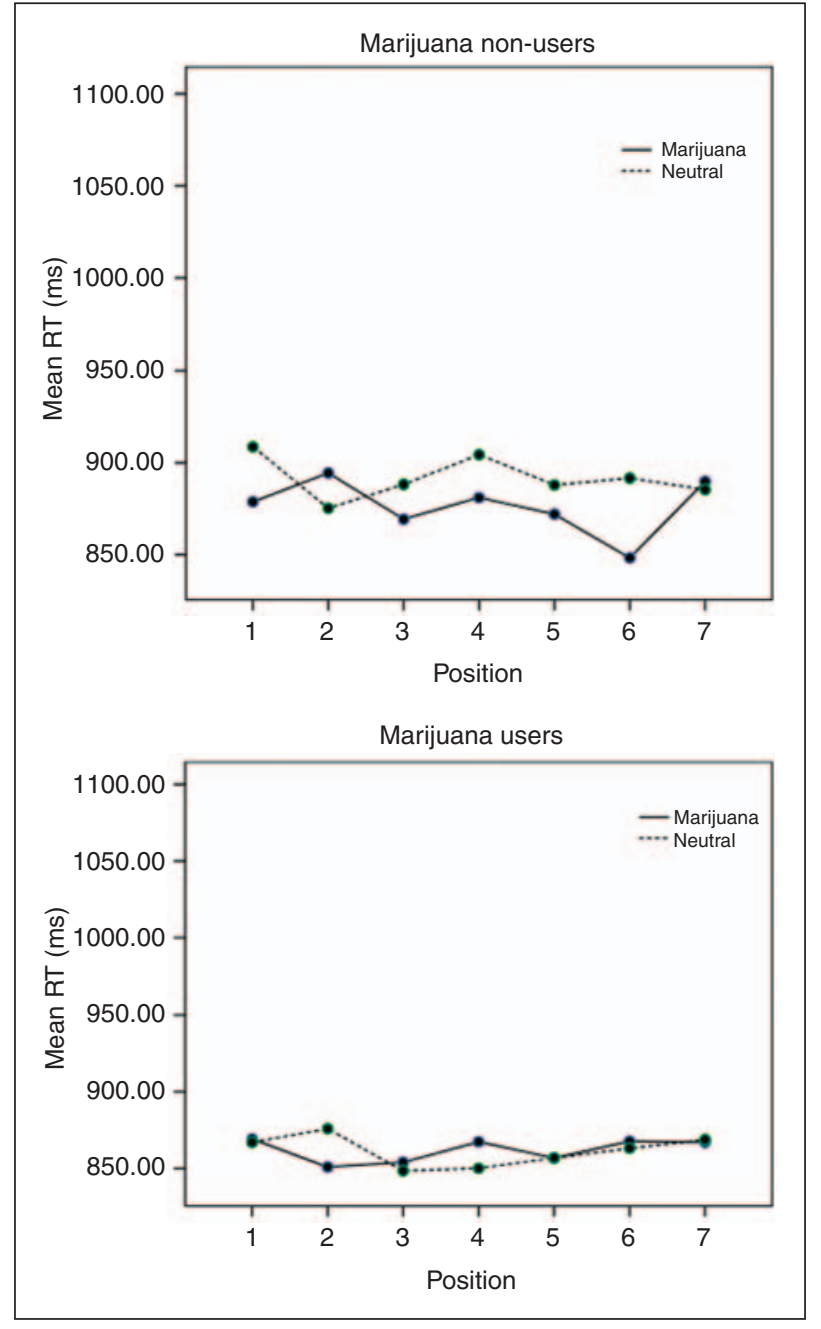

Figure 2. The response latencies to respond to the colour of marijuanaassociated non-words and non-marijuana-associated non-words in the addiction Stroop task.

non-words $(3.2 \%)$ at only position 3 . It is not clear why this difference appeared at position 3 .

\section{Analysis of final memory test}

As there was no evidence of any interference on the addiction Stroop task, it might be argued that the associations formed during the learning phase may have been forgotten during the addiction Stroop task. However, this seems unlikely as a subsequent memory test after the Stroop task showed that participants were still able to classify the non-words as associated to the marijuana or non-marijuana pictures. A Mixed ANOVA with Group and Non-word type showed no significant main or interaction effects (all $F<1.62, p>0.2$ ).

The mean correct retest scores were $8.78(S E=0.38)$ for the marijuana users group and $9.32(S E=0.17)$ for the nonusers group. These mean scores (maximum of 10) were significantly greater than chance (score of five), both $t$ values $>9$, $p<0.001$, indicating that both groups had learned and could 
still remember the association between the images and the non-words after completing the addiction Stroop task.

\section{Experiment 2 Introduction}

Experiment 1 did not produce significant interference from marijuana-associated non-words. In Experiment 2 we investigated a different group that we thought might show a stronger effect, namely, those who had previously administered cocaine. Previous research has shown that cocaine addicts exhibit increased response latencies in tests of response inhibition (Verdejo-Garcia et al., 2007). A few studies have also shown attentional biases to cocaine-related words and pictures (e.g. Hester et al., 2006) indicating a response-selection deficit resulting from an impaired ability to suppress irrelevant information. More importantly, Carpenter et al. (2006) directly compared marijuana and cocaine words in an addiction Stroop task and showed larger interference from cocainerelated words than from marijuana-related words.

The associative-learning technique used in the present study, in which addiction-related pictures are associated to non-words, is particularly appealing not only because past research into drug addiction using associative learning has demonstrated that neutral cues can be conditioned to elicit cravings (Dols et al., 2000), but also because cocaine addicts are particularly susceptible to visual stimuli, often reporting intrusive imagery as being problematic during periods of abstinence (Kavanagh et al., 2004). Experiment 2 therefore aimed to test whether non-words that have been associated to cocaine-related images (as compared with non-cocainerelated images) could subsequently interfere in an addiction Stroop task in a group of cocaine users.

\section{Method \\ Participants}

Sixteen participants from the general public who had previously self-administered crack-cocaine volunteered to take part in the study. The participants were 13 males and three females between ages of 25 and 41 . They were recruited in Canada from a shelter for the homeless, and from England from two local Narcotics Anonymous Fellowships. All participants reported being in abstinence from crack-cocaine for a minimum of one month, with a mean abstinence time of 14 months, with a range of one month to 48 months. However, this was not medically validated.

\section{Design}

The design formed a $2 \times 7$ within-subjects design, with nonword type (cocaine associated, non-cocaine associated) and Position (1-7) as the within-subject factors.

\section{Materials}

The pictures used for this experiment are shown in Figure 3. All images were created with a Fuji film FinePix digital

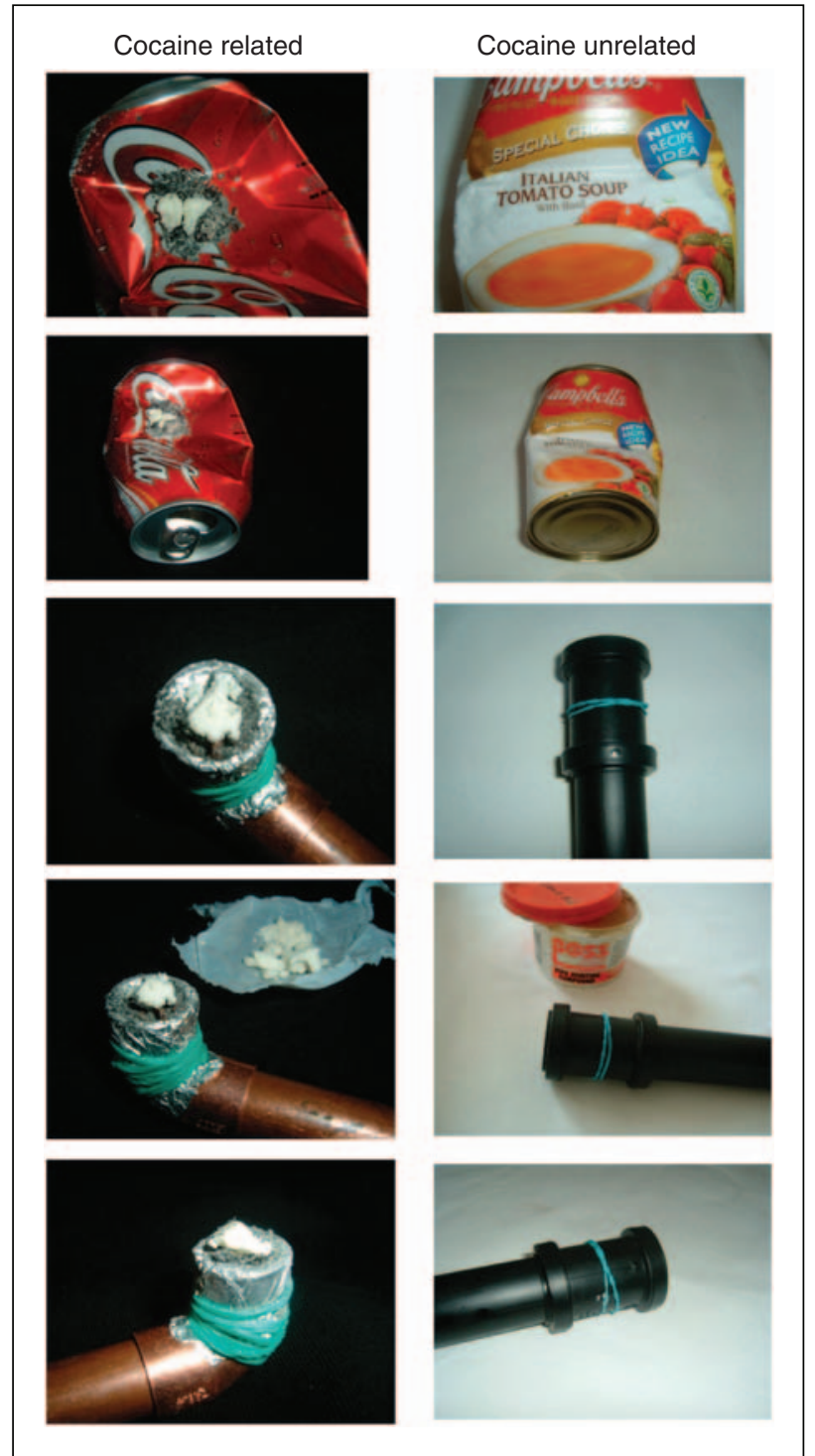

Figure 3. Pictures used to condition non-words in Experiment 2 (cocaine).

camera and were presented in full colour, and appeared 8 inches high and 12 inches wide on the laptop computer screen. The pronounceable non-words and their presentation format, and the e-prime response box, was the same as in Experiment 1. The learning phase, practice phase and Stroop phase were identical to Experiment 1. During the learning phase participants received on average 48.75 trials $(S E=9.83)$ to reach criterion. The number of learning trials participants received in Experiment 2 did not differ significantly from Experiment 1 (compared with marijuana users, $t(41)=0.895, p=0.38$ or non-users $t(39)=0.79, p=0.43)$.

\section{Results}

The RT data was inspected for outliers, and errors were removed using the same criteria as Experiment 1. The main finding in Experiment 2 is that cocaine-associated non-words 


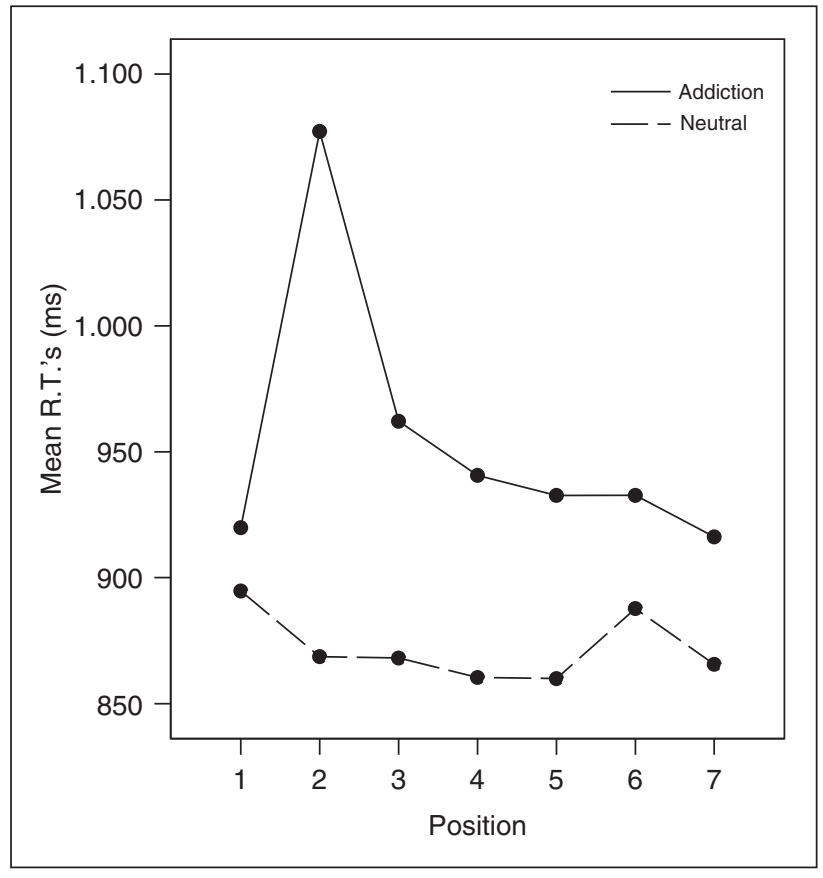

Figure 4. The carryover effect of cocaine-associated non-words and noncocaine-associated non-words in an addiction Stroop task.

produce larger interference in the addiction Stroop task. ANOVA revealed a main effect of Non-word Type: $F(1,15)=4.74 ; \quad M S E=80084, \quad p<0.05$, indicating longer response times in the session containing the cocaine-associated non-words (954 ms) than the session containing the non-cocaine-associated non-words $(872 \mathrm{~ms})$. The analysis also revealed carryover effects indicated by a significant interaction between Non-word Type and Position: $F(6,90)=4.087$, $M S E=7125.973, p=0.001$. The general pattern was for slower responding to the cocaine-associated non-words than the non-cocaine-associated non-words that was largest at position $2(208 \mathrm{~ms})$ and then attenuated at later positions (see Figure 4). Simple effects analysis revealed that interference at position $1(25 \mathrm{~ms})$ was not significant $t(15)=0.616$, $p=0.55$. It was significant at position 2 (208 ms): $t(15)=2.447, p=0.027$. The interference averaged across positions 3-7 (69 ms) was significant: $t(15)=2.23, p<0.05$ and was significantly smaller than the interference at position $2(t(15)=2.21, p<0.05))$.

Overall errors were low $(4.7 \%)$. ANOVA on the errors revealed no significant effects. The main effect of Non-word Type and Position were not significant (both $F$ values $<1$.1, $p>0.4)$. The interaction of Non-word Type and Position was also not significant, $F(6,90)=0.845, M S E=0.001, p=0.54$.

\section{Discussion}

We set out to find if marijuana and cocaine users differed in their performance on an addiction Stroop task where the stimuli were non-words associated in a prior learning phase to addiction or non-addiction-related images. We predicted that the non-words that were paired with the addictionrelated images would elicit interference. Our results demonstrate that only cocaine users showed interference when presented with non-words that had been associated with cocaine-related images. In contrast, marijuana users did not show interference from marijuana-associated nonwords. This pattern is consistent with the findings from Carpenter et al. (2006), who have shown interference from cocaine-related words but not marijuana-related words, in groups of marijuana and cocaine users seeking treatment. This is in contrast to other research that demonstrates cognitive biases to marijuana stimuli using the Stroop task (Cane et al., 2009) and other implicit tasks (e.g. implicit memory associations, Ames and Stacy, 1998; Stacy, 1997).

The two experiments that we carried out were identical except for two features, (i) the type of addiction-related picture used for associative learning and (ii) the type of addiction group used. It seems reasonable to assume that these two differences might account for the different pattern of results shown in our two studies. It could be that the incentive properties and/or the subjective craving elicited by cocaine-related pictures are higher than those for marijuana-related stimuli. It is also possible that negative affect played a role particularly if cocaine images induced greater anxiety than marijuana images. For example, if the addiction-related stimuli reminded individuals about the legal status of cocaine and marijuana use in society, then the cocaine-related images might have induced greater anxiety. Another possibility relates to the fact that marijuana is a psychodepressant (Nocerino et al., 2000) whereas cocaine is a psychostimulant (Carrico et al., 2008; Lile, 2006). If, as previous research has shown, drug-related cues can elicit feelings similar to drug usage (Lyvers, 1998) then the psychostimulant properties of cocaine may have induced a state-dependent increase in anxiety. If the interference from cocaine-related pictures are due to anxiety then this would be consistent with the findings of Richards and Blanchette (2004), who demonstrated larger emotional interference in a high anxious group than a low anxious group from non-words that had previously been associated to negative pictures.

The interaction between non-word type and position was also interesting as it suggests that when cocaine users are faced with cues that remind them of their drug use, those cues may take time to exert their effects on the cognitive system. Our findings suggest that cocaine-associated nonwords had little immediate effect (at position 1) but had the largest effect at position 2, and diminished on subsequent trials. These findings are consistent with carryover effects found with smoking-related words (Waters et al., 2003a) and negative emotional words (see McKenna and Sharma, 2004), thus suggesting that the underlying cause of the carryover effect is related to negative emotion. Although Cane et al. (2009) found carryover effects with marijuana words in marijuana users, our first study failed to find carryover effects using non-words that had been associated with marijuana pictures in a group of marijuana users. One possible explanation is that for some addiction groups, using more distally associated conditioned stimuli may reduce the power of those stimuli to have an effect. Another possibility is that participants in Experiment 1 were not abstinent in their drug use, which may have contributed to attenuate the effects. Further research is required to determine the cause of this 
difference. However, in general, our findings emphasize the need for studies using the addiction Stroop task to use a presentation format that will capture the interference effects when those effects operate in a latent manner. Waters et al. (2003a) have suggested that the carryover effect may reflect rumination or difficulty with disengaging or switching attention. These two suggestions are mirrored in a recent model by Field and Cox (2008), who have suggested that attempts to suppress craving and impaired executive cognitive function can moderate attentional bias.

The present study provides further support that drug cues can be easily trained to previously neutral stimuli. Further research is needed to establish why the marijuana group, despite learning an association between drug cues and novel stimuli, did not show transfer of this learning on a subsequent addiction Stroop task. It might be that more intense methods of associative-learning are necessary for some addictionrelated cues to ensure that the conditioning transfers to a different task. One suggestion is to increase the salience of the conditioning by encouraging participants to think back and remember episodes of drug-taking whilst viewing the picture-non-words pairs. Hogarth and Duka (2006) provide some support for this suggestion as they have shown that conscious expectancy for drug use is necessary for orienting attention to the conditioned stimulus.

It is acknowledged that the present study is not without its limitations; firstly, in the cocaine study there was no control group. This leaves open the possibility that a control group may have shown a similar pattern of results as the cocaine group. This would suggest that the nature of the stimuli, rather than the particular groups used, is important for demonstrating a carryover effect. Other limitations are that the sample size was small and some participants conceded to having poly-drug use problems. Furthermore, comorbidity of clinical disorders was not controlled for in either study, and the participants in both groups had a wide range of drug usage intensity and amount of time in recovery.

\section{References}

Albery IP, Sharma D, Niyazi A, Moss AC (2006) Introduction and methodologies. In: Mafano M, Albery IP (eds) Cognition and Addiction. Oxford: OUP.

Ames SL, Stacy AW (1998) Implicit cognition in the prediction of substance use among drug offenders. Psychol Addict Behav 12: 272-281.

Boyer M, Dickerson M (2003) Attentional bias and addictive behaviour: automaticity in a gambling-specific modified Stroop task. Addiction 98: 61-70.

Budney AJ, Radonovich KJ, Higgins ST, Wong CJ (1998) Adults seeking treatment for marijuana dependence: a comparison with cocaine-dependent treatment seekers. Exp Clin Psychopharmacol 6: 419-426.

Cane J, Sharma D, Albery IP (2009) The addiction Stroop task: examining the fast and slow effects of smoking and marijuanarelated cues. J Psychopharmacol 23: 510-519.

Carpenter KM, Schreibe E, Church S, McDowell D (2006) Drug Stroop performance: Relationships with primary substance of use and treatment outcome in a drug-dependent outpatient sample. Addict Behav 31: 174-181.

Carrico AW, Johnson MO, Morin SF, et al. (2008) Stimulant use is associated with immune activation and depleted tryptophan among HIV-positive persons on anti-retroviral therapy. Brain Behav Immun 22: 1257-1262.

Carter BL, Tiffany ST (2001) The cue-availability paradigm: the effects of cigarette availability on cue reactivity in smokers. Exp Clin Psychopharmacol 9: 183-190.

Cox WM, Fadardi JS, Pothos EM (2006) The addiction-Stroop test: Theoretical considerations and procedural recommendations. Psychol Bull 132: 443-476.

Cox WM, Hogan LM, Kristian MR, Race JH (2002) Alcohol attentional bias as a predictor of alcohol abusers' treatment outcome. Drug Alcohol Depend 68: 237-243.

Cox WM, Pothos EM, Hosier SG (2007) Cognitive-motivational predictors of excessive drinkers' success in changing. Psychopharmacology 192: 499-510.

Dols M, Willems B, Van Den Hout M, Bittoun R (2000) Smokers can learn to influence their urge to smoke. Addict Behav 25: 103-108.

Field M, Cox WM (2008) Attentional bias in addictive behaviors: A review of its development, causes, and consequences. Drug Alcohol Depend 97: 1-20.

Field M, Eastwood B, Bradley BP, Mogg K (2006) Selective processing of cannabis cues in regular cannabis users. Drug Alcohol Depend 85: 75-82.

Hester R, Dixon V, Garavan H (2006) A consistent attentional bias for drug-related material in active cocaine users across word and picture versions of the emotional Stroop task. Drug Alcohol Depend 81: 251-257.

Hogarth L, Duka T (2006) Human nicotine conditioning requires explicit contingency knowledge: Is addictive behaviour cognitively mediated? Psychopharmacology 184: 553-566.

Kavanagh DJ, Andrade J, May J (2004) Beating the urge: Implications of research into substance-related desires. Addict Behav 29: 1359-1372.

Larsen RJ, Mercer KA, Balota DA (2006) Lexical characteristics of words used in emotional Stroop experiments. Emotion 6: 62-72.

Lazev AB, Herzog TA, Brandon TH (1999) Classical conditioning of environmental cues to cigarette smoking. Exp Clin Psychopharmacol 7: 56-63.

Lile JA (2006) Pharmacological determinants of the reinforcing effects of psychostimulants: relation to agonist substitution treatment. Exp Clin Psychopharmacol 14: 20-33.

Lyvers M (1998) Drug addiction as a physical disease: the role of physical dependence and other chronic drug-induced neurophysiological changes in compulsive drug self-administration. Exp Clin Psychopharmacol 6: 107-125.

McKenna FP, Sharma D (2004) Reversing the emotional Stroop effect: The role of fast and slow components. J Exp Psychol Learn Mem Cogn 30: 382-392.

Munafo M, Mogg K, Roberts S, Bradley BP, Murphy M (2003) Selective processing of smoking-related cues in current smokers, ex-smokers, and never-smokers on the modified Stroop paradigm. J Psychopharmacol 17: 311-317.

Nocerino E, Amato M, Izzo AA (2000) Cannabis and cannabinoid receptors. Fitoterapia 71: S6-S12.

Perry MJ, Mandell W (1995) Psychosocial factors associated with the initiation of cocaine use among marijuana users. Psychol Addict Behav 9: 91-100.

Phaf RH, Kan KJ (2007) The automaticity of emotional Stroop: A meta-analysis. J Behav Ther Exp Psychiatry 38: 184-199.

Rastle K, Harrington J, Coltheart M (2002) 358,534 nonwords: The ARC Nonword Database. $Q J$ Exp Psychol 55A: 1339-1362.

Richards A, Blanchette I (2004) Independent manipulation of emotion in an emotional Stroop task using classical conditioning. Emotion 4: 275-281.

Robinson TE, Berridge KC (1993) The neural basis of drug craving: an incentive-sensitization theory of addiction. Brain Res Rev 18: 247-291. 
Sharma D, Albery IP, Cook C (2001) Selective attentional bias to alcohol related stimuli in problem drinkers and non problem drinkers. Addiction 96: 285-295.

Stacy AW (1997) Memory activation and expectancy as prospective predictors of alcohol and marijuana use. J Abnorm Psychol 106: 61-73.

Stroop JR (1935) Studies of interference in serial verbal reactions. $J$ Exp Psychol 18: 643-661.

Verdejo-Garcia AJ, Perales JC, Perez-Garcia M (2007) Cognitive impulsivity in cocaine an heroine polysubstance abusers. Addict Behav 32: 950-966.
Waters AJ, Sayette MA, Franken IHA, Schwartz JE (2005) Generalizability of carry-over effects in the emotional Stroop task. Behav Res Ther 43: 715-732.

Waters AJ, Sayette MA, Wertz J (2003a) Carry-over effects can modulate emotional Stroop effects. Cogn Emot 17: 501-509.

Waters AJ, Shiffman S, Sayette MA, Paty JA, Gwaltney CJ, Balabanis MH (2003b) Attentional bias predicts outcome in smoking cessation. Health Psychol 22: 378-387. 